\title{
Adaptive Predictive Functional Control WITH SIMILAR PI STRUCTURE USING UNSTRUCTURED SYSTEM IDENTIFICATION BASED ON LAGUERRE FUNCTIONS
}

\author{
Reza Dadkhah Tehrani ${ }^{1}$ and Mohammad Hossein Ferdowsi ${ }^{2}$ \\ ${ }^{1}$ Department of Control Engineering, Malek Ashtar University of Technology, Tehran, \\ Iran \\ r.dadkhah1388@gmail.com \\ 2 Department of Control Engineering, Malek Ashtar University of Technology, Tehran, \\ Iran \\ ferdowsi@mut.ac.ir
}

\begin{abstract}
In this paper, unstructured system identification algorithm based on orthonormal Laguerre functions is combined with predictive functional control such that similar classical PI controller is constructed. Lack of mathematical model and initial information about process is not a restriction for mentioned algorithm and unstructured system identification based on Laguerre functions can overcome these restrictions. Augmenting new state variables to system state space, a new algorithm is constructed. This algorithm has similar structure with classical PI controller and in predictive control's cost function, in addition to tracking error, system states is utilized, that leads to improve controller dynamical performance. This new algorithm is simulated on the superheated steam temperature system in thermal power plant. Simulation results show capabilities of this algorithm.
\end{abstract}

\section{KEYWORDS}

Predictive Functional Control, Laguerre Functions, Unstructured System Identification, Superheated Steam Temperature, PI Controller.

\section{INTRODUCTION}

Orthonormal functions, due to its similarity to Pade approximate, has many advantages in identification of control systems with variable time-delay, unknown order and structural parameters. Moreover, combining these functions with Volterra series, this model can be easily used to the field of nonlinear predictive control [1]. Therefore, these models are more applicable to process control than other traditional structured system identifications such as ARMA model. Laguerre series is one of the most famous of orthonormal functions that for first time introduced by French mathematician- Laguerre, in 1879.

Laplace transforms of Laguerre series that the first time described by Lee in 1931, can be used in approximating linear system model. In 1995, Heuberger and Wahlberg proposed methods on 
approximating dynamic linear systems by orthonormal functions in Z-domain and S-domain, respectively [1].

Predictive Functional Control (PFC) belong to a class of digital control methods called ModelBased Predictive Control (MBPC) and for the first time introduced and implemented by Richalet and Kuntze successfully at the end of the 1980's. Less calculation and high control precision are most important advantages of this algorithm; hence, this method is very suitable for applications need high speed. Control effort is obtained by minimization of difference between predicted model output and reference trajectory on the finite horizon time. Moreover, this algorithm is robust with respect to modeling errors [2]. However, this method would be out of work when the model changed greatly.

In this paper, a new control method is proposed that combines predictive functional control with unstructured system identification methods based on Laguerre functions. On the other hand, by selecting the state space model of process based on Laguerre functions, inner states of system can be easily described and these states can be used in controller design. If these states contain some physical variables, prediction of their future changes can give important information regarding the processes [3]. However, the classical PFC algorithm has not made full use of these merits.

On the other hand, the superheater is an important part of the steam generation process in the boiler-turbine system, where steam is superheated before entering the turbine that drives the generator. Not only the steam generation process is highly nonlinear, the temperature and the pressure in the superheater are extremely high. Therefore, controlling superheated steam temperature is not only technically challenging, but also economically important [4]. The main steam temperature object of power plant is a complex and time-varying system with large delay and inertia. It is very difficult to get satisfied control result by using traditional control strategy [5]. In the recent years, advance control algorithms such as Neuro-fuzzy GPC [4], Gray predictive adaptive Smith-PID [5] and Fuzzy-Immune PID [6] use for control of this system.

New PFC algorithm in this paper uses system states in controller design. The structure of this controller is similar to classical PI controller, but contains a P-step set point feedforward control ( $\mathrm{P}$ is the prediction horizon.).

In section (2) of this paper, we will describe system identification is done with Laguerre functions. Section (3) will present how the state space of system is extended. Section (4) represents control law and in the follow, in section (5), simulations on the superheated steam temperature system for verification of controller performance are represented. In the end, in section (6), conclusion of the paper is presented.

\section{SYSTEM IDENTIFICATION BASED ON LAGUERRE FUNCTIONS}

Laguerre functions, a complete orthonormal set in $\mathrm{L}_{2}(0, \infty)$, have been used often because of their convenient network realization and their similarity. These functions are defined as a functional series

$\Phi_{i}(t)=\sqrt{2 p} \frac{e^{p t}}{(i-1) !} \frac{d^{i-1}}{d t^{i-1}}\left[t^{i-1} \cdot e^{2 p t}\right], i=1,2, \ldots, \infty$

Where $p$ is a constant called time scaling factor, and $t \in[0, \infty)$ is a time variable. The Ztransform of the Laguerre functions is [7]: 
International Journal of Control Theory and Computer Modelling (IJCTCM) Vol.2, No.5, September 2012

$\Phi_{i}(z)=Z\left\{\Phi_{i}(t)\right\}=\frac{\sqrt{1-p^{2}}}{1-p z^{-1}}\left(\frac{z^{-1}-p}{1-p z^{-1}}\right)^{i-1}, \quad i=1,2, \ldots, \infty$

Any open-loop stable system can be approximated by $n$ order Laguerre series as shown in figure 1 .

$Y_{m}(z)=\sum_{i=1}^{n} C_{i} \Phi_{i}(z) U(z)=\sum_{i=1}^{n} C_{i} l_{i}(z)$

There are several ways to express the Laguerre ladder network, but for our purpose, it is convenient to represent it in a state space form. Sate space expression with Laguerre functions after discretization is

$\bar{X}(k+1)=\bar{A} \bar{X}(k)+\bar{B} u(k-d)$

$\bar{y}(k)=\bar{C} \bar{X}(k)$

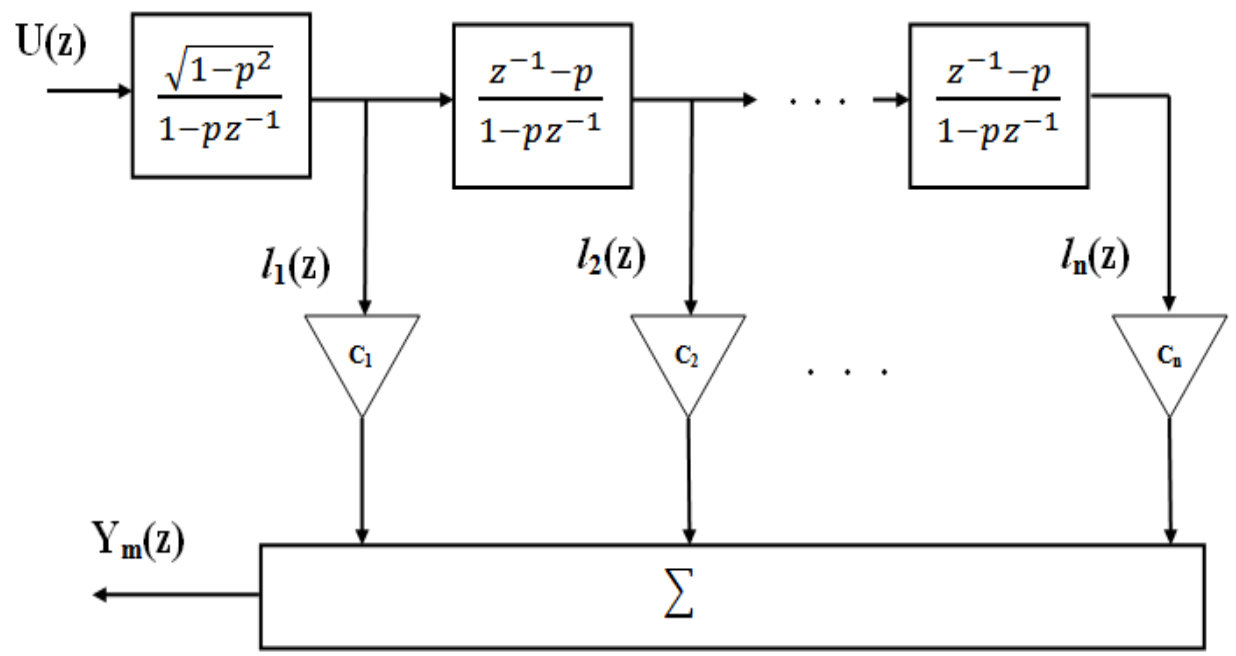

Figure 1. Discrete Laguerre network structure

Where $\bar{X}(k)$ is the state vector with dimension $n \times 1$ and $\mathrm{u}(\mathrm{k})$ is system input. $\bar{A}$ is a lower triangular $n \times n$ matrix. $\bar{B}$ is the coefficient vector of the control input with dimension $n \times 1$. Elements of $\bar{A}$ and $\bar{B}$ are determined off-line, hence reduce the computational burden greatly. $d$ is delay time of the system. If $T$ is the sampling period and

$\tau_{1}=e^{-p T}, \quad \tau_{2}=T+\frac{2}{p}\left(e^{-p T}-1\right), \quad \tau_{3}=-T e^{-p T}-\frac{2}{p}\left(e^{-p T}-1\right)$

$\tau_{4}=\sqrt{2 p} \frac{\left(1-\tau_{1}\right)}{p}, \quad \alpha=\tau_{1} \tau_{2}+\tau_{3}$

then $\bar{A}$ and $\bar{B}$ matrices are expressed as 
International Journal of Control Theory and Computer Modelling (IJCTCM) Vol.2, No.5, September 2012

$\bar{A}=\left[\begin{array}{cccc}\tau_{1} & 0 & \cdots & 0 \\ -\frac{\alpha}{T} & \tau_{1} & \cdots & 0 \\ \vdots & \vdots & \ddots & \vdots \\ \frac{(-1)^{n-1} \tau_{2}^{n-2} \alpha}{T^{n-1}} & \cdots & -\frac{\alpha}{T} & \tau_{1}\end{array}\right], \quad \bar{B}=\left[\tau_{1}\left(\frac{-\tau_{2}}{T}\right) \tau_{4} \cdots\left(\frac{-\tau_{2}}{T}\right)^{n-1} \tau_{4}\right]^{T}$

in addition, state vector is [2]

$\bar{X}(k)=\left[\begin{array}{llll}\bar{x}_{1}(k) \bar{x}_{2}(k) & \cdots & \bar{x}_{n}(k)\end{array}\right]^{T}$

$\bar{C}$ is the observer coefficient vector with dimension $n \times 1$ and for describing relation between Laguerre model and process, which can be identified online by RLS (Recursive Least Square) algorithm. So

$\bar{C}(k)=\bar{C}(k-1)+\left\{M(k)[\bar{y}(k)-\bar{C}(k-1) \bar{X}(k)\}^{T}\right.$

$M(k)=\frac{P(k-1) \bar{X}(k)}{\lambda+\bar{X}^{T}(k) P(k-1) \bar{X}(k)}$

$P(k)=\frac{1}{\lambda}\left[P(k-1)-M(k) \bar{X}^{T}(k) P(k-1)\right]$

where $0<\lambda \leq 1, \lambda$ is the forgetting factor

$\bar{C}$ is assumed bounded, system describes in Eq. (4) is stable, observable and controllable. Moreover, we assume that Laguerre functions are an exact description of the system. With these assumptions, adaptive PFC algorithm with similar classical PI controller structureis presented.

\section{EXTENDED STATE SPACE SYSTEM REPRESENTATION}

By augmenting $\mathrm{u}(\mathrm{k}-1), \mathrm{u}(\mathrm{k}-2), \ldots, \mathrm{u}(\mathrm{k}-\mathrm{d})$ as the system's new state variables and $\mathrm{u}(\mathrm{k})$ as the input, new state space equation of system is as

$X(k+1)=A X(k)+B u(k)$

$y(k)=C X(k)$

Where

$A=\left[\begin{array}{cccccc}\bar{A} & \bar{B} & \underline{O} & \underline{O} & \cdots & \underline{O} \\ \bar{O} & 0 & 1 & 0 & \ldots & 0 \\ \vdots & \vdots & & \ddots & & \vdots \\ \bar{O} & 0 & & 0 & & 1 \\ \bar{O} & 0 & & \ldots & & 0\end{array}\right], \quad B=\left[\underline{O}^{T}, 0, \ldots, 0,1\right]^{T}, \quad C=[\bar{C}, 0,0, \ldots, 0]$

$X(k)=\left[\bar{X}(k)^{T}, u(k-d), u(k-d+1), \ldots, u(k-1)\right]^{T}$

$\overline{\boldsymbol{O}}$ and $\underline{\boldsymbol{O}}$ is the zero vectors with dimensions $n \times 1$ and $n \times 1$, respectively. Now, for import integral mechanism, which canguarantee zero steady-state error in the closed-loop system, using different operator, we can rewrite the state space system's equation as below: 
$\Delta X(k+1)=A \Delta X(k)+B u(k)-B u(k-1)$

$y(k)=C X(k)$

Where $\Delta$ is the difference operator $\left(\Delta=1-z^{-1}\right)$.

Regarding $\mathrm{r}(\mathrm{k})$ as the input reference trajectory, the output tracking error is defined as

$e(k)=y(k)-r(k)$

Combining Eq. (11) and Eq. (12), the output error in the next step is

$e(k+1)=e(k)+C A \Delta X(k)+C B u(k)-C B u(k-1)-\Delta r(k+1)$

Augmenting output-tracking error to state, state equations become

$z(k+1)=\tilde{A} z(k)+\tilde{B} u(k)-\tilde{B} u(k-1)+\tilde{C} \Delta r(k+1)$

Where

$z(k)=\left[\begin{array}{c}e(k) \\ \Delta X(k)\end{array}\right], \quad \tilde{A}=\left[\begin{array}{cc}1 & C A \\ \tilde{O} & A\end{array}\right], \tilde{B}=\left[\begin{array}{c}C B \\ B\end{array}\right], \tilde{C}=\left[\begin{array}{c}-1 \\ \tilde{O}\end{array}\right]$

Where $\tilde{O}$ is the zero vector with dimension $(n+d) \times 1$. Eq. (14) is used to design a novel predictive functional controller.

\section{PFC CONTROL ALGORITHM}

\subsection{Cost FUnCTION}

Consider the following optimal problem over a certain horizon $P[3]$ :

$J=\frac{1}{2} \sum_{j=1}^{P} z^{T}(k+j) Q_{j} z(k+j)$

where $P$ is the number of fitting points and $Q_{j}$ is the symmetrical weighted matrix with dimension $(n+d+1) \times(n+d+1)$ and generally

$Q_{j}=\operatorname{diag}\left\{q_{j 1}, q_{j 2}, \ldots, q_{j(n+1)}, 0, \ldots, 0\right\}$

For selecting elements of weighted matrix, $Q_{j}$, we can say:

- Usually, $q_{j l} \neq 0$, because the output tracking error must be considered in the cost function. $q_{j 2}, \ldots, q_{j(n+1)}$ must be adjusted to regulate the system's state vector increments $\Delta \bar{X}(k)$.

- By selecting $Q_{j}=0, j<N_{P}$ optimizing time starts at time $k+N_{P}$ and this means the effect of the system's dead time is considered.

- Considering $N_{P}=P$ indicates only one point prediction. 
- $Q_{j}=\operatorname{diag}\left\{q_{j 1}, 0, \ldots, 0\right\}$, indicates that only output errors are weighted; this method is equivalent to the traditional predictive functional control method.

\subsection{STATE PREDICTION AND CONTROLLER DESIGN}

PFC has the same principle as the classical predictive control strategy and uses a model to predict future process output.However, in PFC algorithm, for reducing calculation burden, control law is considered as a linear combination of a set of base functions. In this method, only it is necessary to calculate the weight coefficients of base functions of linear combination. Choice of base functions is related to the nature of the process and characteristic of the reference trajectory. Therefore, the control law can be defined as:

$u(k+i)=\sum_{j=1}^{N} \mu_{j} f_{j}(i)$

Where $\mu_{j}$ are the coefficients of base functions and $f_{j}(i)$ are the values of the base functions in time $i$. $\mathrm{N}$ is the number of the base functions. For example, if $N=2$, means base functions are step and ramp. In this case, control law is:

$u(k+i)=\mu_{1}(\mathrm{k})+\mu_{2}(\mathrm{k}) \times i$

PFC algorithm has to find a set of future control variables to make the future outputs of the process as close as possible to the reference trajectory.

Combining Eq. (14) and Eq. (18), the future prediction of the system is

$$
\begin{aligned}
& z(k+1)=\tilde{A} z(k)+\tilde{B} \sum_{j=1}^{N} \mu_{j} f_{j}(0)-\tilde{B} u(k-1)+\tilde{C} \Delta r(k+1) \\
& z(k+2)=\tilde{A}^{2} z(k) \\
& \quad+\tilde{A} \tilde{B} \sum_{j=1}^{N} \mu_{j} f_{j}(0)-\tilde{A} \tilde{B} u(k-1) \\
& \quad+\widetilde{B} \sum_{j=1}^{N} \mu_{j} f_{j}(1)-\widetilde{B} \sum_{j=1}^{N} \mu_{j} f_{j}(0)+\tilde{A} \tilde{C} \Delta r(k+1)+\tilde{C} \Delta r(k+2)=\tilde{A}^{2} z(k) \\
& \quad+(\tilde{A} \tilde{B}-\tilde{B}) \sum_{j=1}^{N} \mu_{j} f_{j}(0)+\widetilde{B} \sum_{j=1}^{N} \mu_{j} f_{j}(1)-\tilde{A} \tilde{B} u(k-1)+\tilde{A} \tilde{C} \Delta r(k+1) \\
& +\tilde{C} \Delta r(k+2)
\end{aligned}
$$


International Journal of Control Theory and Computer Modelling (IJCTCM) Vol.2, No.5, September 2012

$$
\begin{aligned}
& z(k+P)=\tilde{A}^{P} z(k) \\
& \quad+\tilde{A}^{P-1} \tilde{B} \sum_{j=1}^{N} \mu_{j} f_{j}(0)-\tilde{A}^{P-1} \tilde{B} u(k-1)+\tilde{A}^{P-2} \tilde{B}\left[\sum_{j=1}^{N} \mu_{j} f_{j}(1)-\sum_{j=1}^{N} \mu_{j} f_{j}(0)\right] \\
& \quad+\cdots+\tilde{B}\left[\sum_{j=1}^{N} \mu_{j} f_{j}(P-1)-\sum_{j=1}^{N} \mu_{j} f_{j}(P-2)\right]+\tilde{A}^{P-1} \tilde{C} \Delta r(k+1)+\cdots \\
& \quad+\tilde{C} \Delta r(k+P) \\
& =\tilde{A}^{P} z(k)+\left(\tilde{A}^{P-1} \tilde{B}-\tilde{A}^{P-2} \tilde{B}\right) \sum_{j=1}^{N} \mu_{j} f_{j}(0)+\cdots+(\tilde{A} \tilde{B}-\tilde{B}) \sum_{j=1}^{N} \mu_{j} f_{j}(P-2) \\
& \quad+\tilde{B} \sum_{j=1}^{N} \mu_{j} f_{j}(P-1)-\tilde{A}^{P-1} \tilde{B} u(k-1)+\tilde{A}^{P-1} \tilde{C} \Delta r(k+1)+\cdots+\tilde{C} \Delta r(k+P)
\end{aligned}
$$

This can be written in the vector form as

$Z=F Z(k)-\bar{G} u(k-1)+G U+S \Delta R$

Where, $Z, F, \bar{G}, G, U, S$ and $\Delta R$ represented in the appendix.

Now, we can rewrite the cost function as:

$J=\frac{1}{2} Z^{T} Q Z$

Where

$Q=$ Block diag $\left\{Q_{1}, Q_{2}, \ldots, Q_{P}\right\}$

The optimal solution of the cost function is:

$U=-\left(G^{T} Q G\right)^{-1} G^{T} Q[F z(k)-\bar{G} u(k-1)+S \Delta R]$

Therefore, the weighting coefficient of control law is:

$$
\begin{gathered}
\begin{array}{c}
\mu_{1}=-(1,0, \ldots, 0)\left(G^{T} Q G\right)^{-1} G^{T} Q[F z(k)-\bar{G} u(k-1)+S \Delta R] \\
=-h_{1} z(k)+h_{u 1} u(k-1)-m_{1} \Delta R
\end{array} \\
\begin{array}{c}
\mu_{2}=-(0,1, \ldots, 0)\left(G^{T} Q G\right)^{-1} G^{T} Q[F z(k)-\bar{G} u(k-1)+S \Delta R] \\
=-h_{2} z(k)+h_{u 2} u(k-1)-m_{2} \Delta R
\end{array} \\
\vdots \\
\mu_{N}=-(0,0, \ldots, 1)\left(G^{T} Q G\right)^{-1} G^{T} Q[F z(k)-\bar{G} u(k-1)+S \Delta R] \\
=-h_{N} z(k)+h_{u N} u(k-1)-m_{N} \Delta R
\end{gathered}
$$

then the current control input is 


$$
\begin{aligned}
u(k)=\sum_{j=1}^{N} \mu_{j} & f_{j}(0) \\
& =f_{1}(0)\left[-h_{1} z(k)+h_{u 1} u(k-1)-m_{1} \Delta R\right] \\
& +f_{2}(0)\left[-h_{2} z(k)+h_{u 2} u(k-1)-m_{2} \Delta R\right]+\cdots \\
& +f_{N}(0)\left[-h_{N} z(k)+h_{u N} u(k-1)-m_{N} \Delta R\right] \\
& =-\sum_{i=1}^{N} f_{i}(0) h_{i} z(k)+\sum_{i=1}^{N} f_{i}(0) h_{u i} u(k-1)-\sum_{i=1}^{N} f_{i}(0) m_{i} \Delta R \\
& =-H z(k)+H_{u} u(k-1)-M \Delta R
\end{aligned}
$$

Where

$H=\sum_{i=1}^{N} f_{i}(0) h_{i}, \quad H_{u}=\sum_{i=1}^{N} f_{i}(0) h_{u i}, \quad M=\sum_{i=1}^{N} f_{i}(0) m_{i}$

Defining

$H=\left(K_{e}, K_{x_{1}}, \ldots, K_{x_{n}}, K_{d}, \ldots, K_{1}\right), \quad M=\left(l_{1}, l_{2}, \ldots, l_{P}\right)$

Combining Eq. (23) and Eq. (25), we can write the control law as:

$u(k)=-K_{e} e(k)-\sum_{j=1}^{n} K_{x j} \Delta \bar{x}_{j}(k)-\sum_{j=1}^{d} K_{j} \Delta u(k-j)+H_{u} u(k-1)-\sum_{j=1}^{P} l_{j} \Delta r(k+j)$

It is seen that the control input includes:

(a) Tracking error's feedback, (b) Feedback of system states, (c) Feedback of past inputs and d) Feedforward of P-step set points.

The tracking error's feedback, deletes the tracking error, the feedback of states and past input's improves the dynamical performance of the system, and the feedforward of P-step set points improves the system's tracking performance. This control structure is similar to classical PI controller, with additional P-step feedforward term. This new algorithm is designated as similar PI structure predictive functional control.

\section{SimUlations}

In this section, the performance of predictive functional control algorithm by simulation is evaluated and effect of the free parameters of the controller on controller performance is discussed. This controller has similar PI structure based on system identification using Laguerre functions, which is suitable for process identification with no certain structure and are time varying. In the termal power plant, when a unit's output load was $180 \mathrm{MW}$, the characteristic of the main steam temperature object is [5]:

$G(s)=\frac{9 e^{-12 s}}{(1+26 s)(1+32 s)}$

The discrete transfer function of system with sampling time $T_{s}=3 \mathrm{sec}$. is 
$G(z)=\frac{0.04541+0.04236 z^{-1}}{1-1.802 z^{-1}+0.8113 z^{-2}} z^{-5}$

at $450 \mathrm{~s}$, the object changed as [5]:

$G(s)=\frac{7 e^{-6 s}}{(1+22 s)(1+28 s)}$

that in discrete form is:

$G(z)=\frac{0.04717+0.04349 z^{-1}}{1-1.771 z^{-1}+0.7839 z^{-2}} z^{-3}$

The setting value of main steam temperature system has a step change in $900 \mathrm{~s}$ and changed from 1 to 1.4 .

From figure 2, the objective is to control the superheated steam temperature by controlling the flow of spray water using the spray water valves. Water is sprayed onto the steam to control the superheated steam temperature in both the low and high temperature superheaters. It is undesirable that the steam temperature is too high, as it can damage the superheater and the high pressure turbine, or too low, as it will lower the efficiency of the power plant.

Assume that there is no certain process model. As in ebove mentioned, this algorithm can identify system without initial information from process and it will be adapted with varying process.

The free parameters of the controller are selected as:

$\lambda=0.99, n=4, T=15, p=0.0095, N=3, P=20, d=5$

$Q_{j}=\operatorname{diag}\{100,0,0,0,0,0,0,0,0,0\}$

It should be noted that when $Q_{j}$ is chosen as $Q_{j}=\operatorname{diag}\{100,0,0,0,0,0,0,0,0,0\}$, only the tracking error is in the cost function. Therefore, this method is equivalent to classical PFC and cost function does not include states of the system.

Free parameters are selected as (31), and the closed loop system output is shown in figure 3. In figure 4 , control effort and its factors is shown.

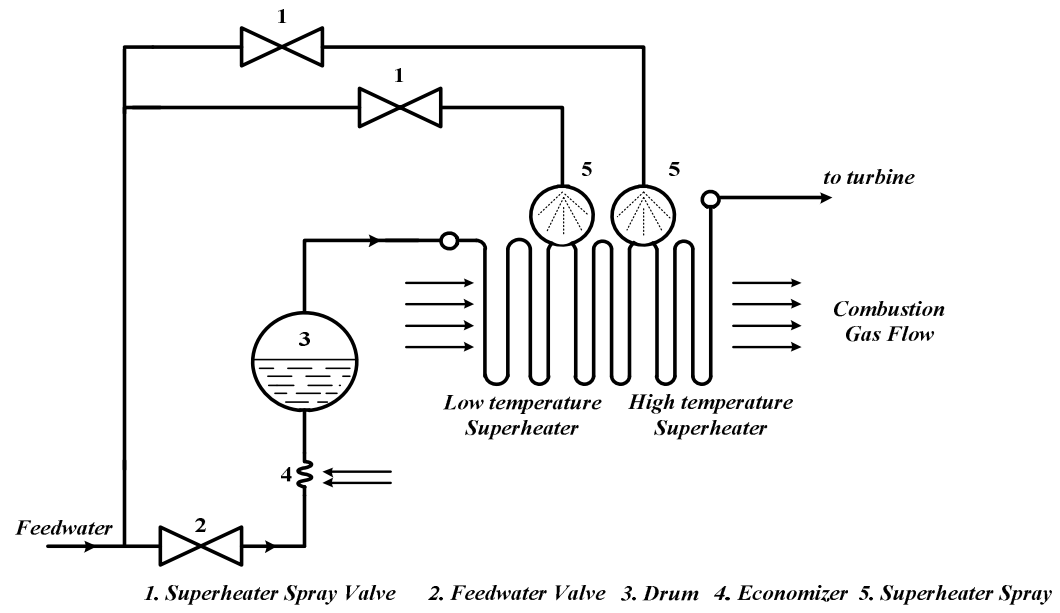

Figure 2. Superheater steam generation process 
From figure 3 and 4, it is evident we have high performance tracker which is robust to variations of process. However, in the response of system, there exists overshoots that not desired in the most applications and it can damage the superheater and the high pressure turbine. Choosing weighting matrix $Q_{j}$ as $Q_{j}=\operatorname{diag}\{100,0,10,10,0,0,0,0,0,0\}$, overshoots of system response reduced effectively and tracking error is very small. In the other word, with entering the states of system in the cost function, the system performance is controllable, and desired specifications are fulfilled. This is an important advantage of new control algorithm. In figure 5, system response with $Q_{j}=\operatorname{diag}\{100,0,10,10,0,0,0,0,0,0\}$ is shown. In Figure 6, control effort and its factors for this case is shown. The variations of control effort are lower and smoother than previous case. This prevents the saturation of the system actuators.

\section{Conclusions}

Presented adaptive PFC algorithm with similar PI structure using unstructured system identification based on Laguerre function, has high performance in processes having no specified model. This algorithm can improve dynamical performance of the system in comparison with classical PFC. Proposed approach has been successfully applied to simulated superheater model of the power plant. Better performance is obtained from proposed algorithm than from the classical PFC controller in controlling the superheatead steam temperature under large load changes. The simulation results show that the present control algorithm can effectively overcome the object parameters and model change, with the character of small overshoot, fast regulating speed and strong robustness. It has a good control performance to the main steam temperature system. The simulation results illustrated that the proposed algorithms lead to excellent control performance and high robustness.

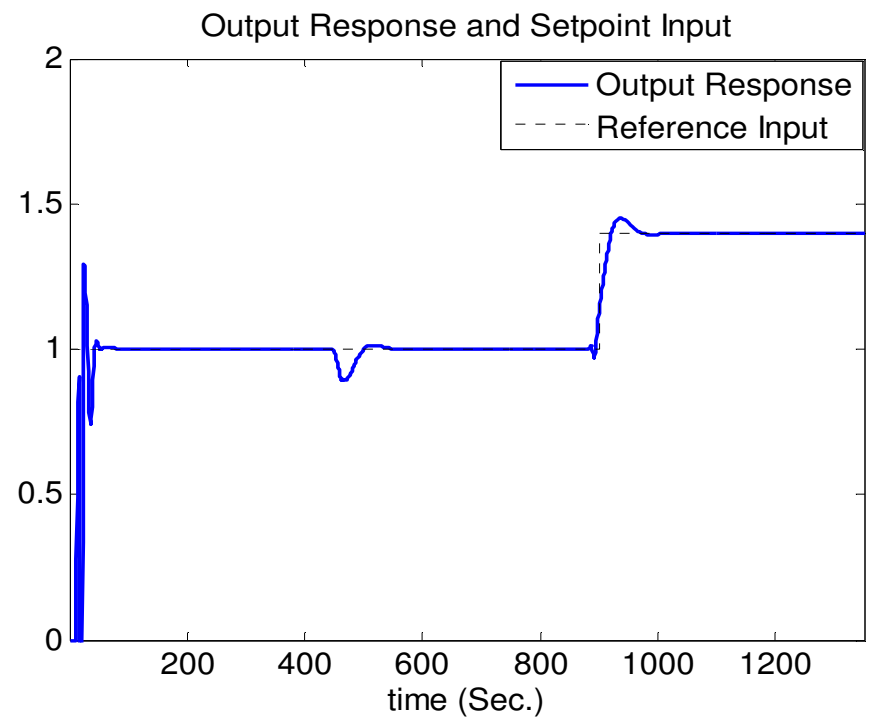

Figure 3. Closed loop system response with $\mathrm{Qj}=\operatorname{diag}\{100,0,0,0,0,0,0,0,0,0\}$ 

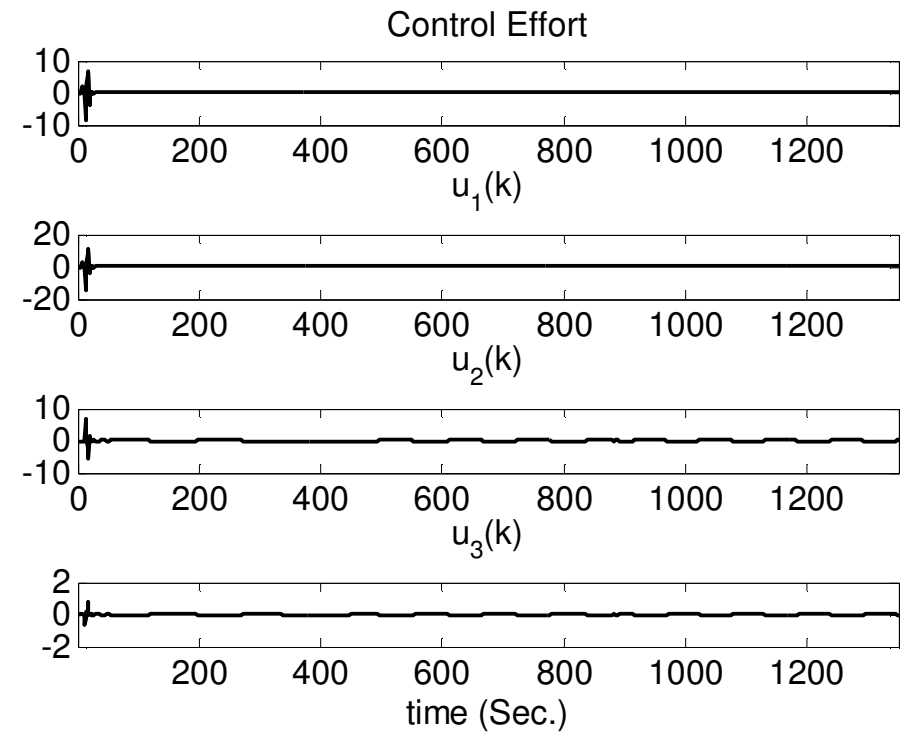

Figure 4. Control effort and its factors with $\mathrm{Qj}=\operatorname{diag}\{100,0,0,0,0,0,0,0,0,0\}$

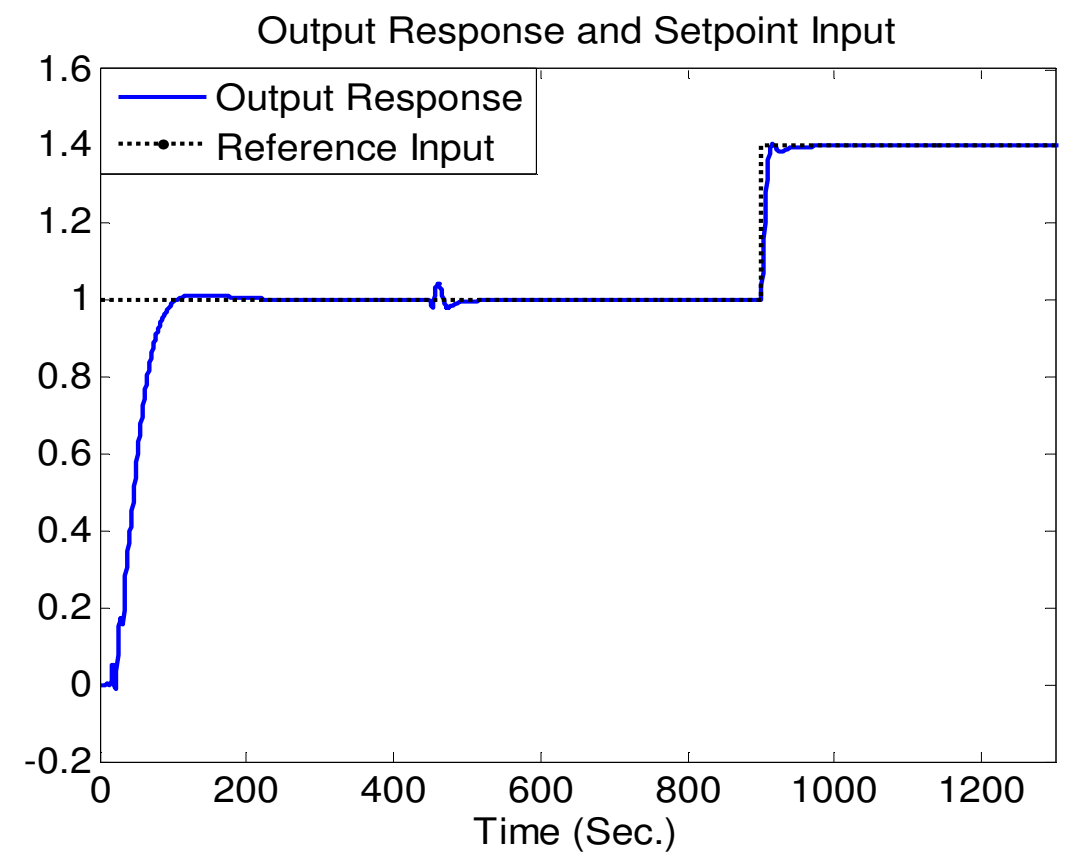

Figure 5. close loop system response with $\mathrm{Qj}=\operatorname{diag}\{100,0,10,10,0,0,0,0,0,0\}$ 

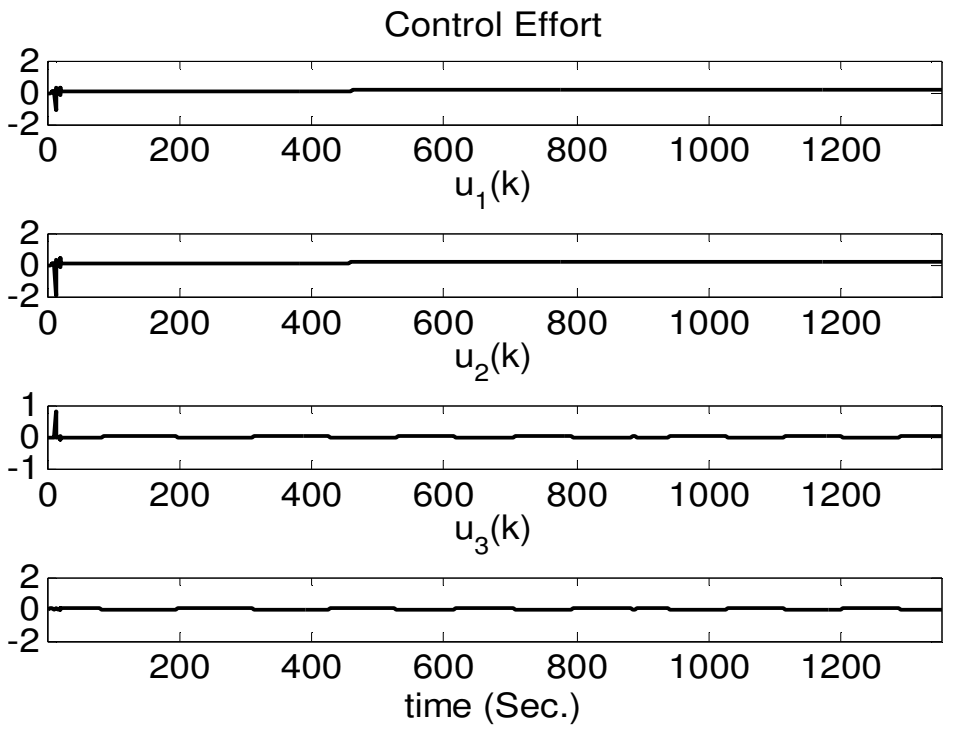

Figure 6. Control effort in the case of $\mathrm{Qj}=\operatorname{diag}\{100,0,10,10,0,0,0,0,0,0\}$

\section{APPENDIX}

$$
\begin{array}{rl}
Z=[z(k+1), z(k+2), \ldots, z(k+P)]^{T} & F=\left[\tilde{A}^{T}, \tilde{A}^{2}, \ldots, \tilde{A}^{P^{T}}\right]^{T} \\
\bar{G}=\left[\tilde{B}^{T},(\tilde{A} \tilde{B})^{T}, \ldots,\left(\tilde{A}^{P-1} \tilde{B}\right)^{T}\right]^{T} & U=\left[\mu_{1}, \mu_{2}, \ldots, \mu_{N}\right]^{T} \\
\Delta R=[\Delta r(k+1), \Delta r(k+2), \ldots, \Delta r(k+P)]^{T} & S=\left[\begin{array}{cccc}
\tilde{C} & \ldots & 0 \\
\tilde{A} \tilde{C} & \tilde{C} & & \\
\vdots & \vdots & \ddots & \vdots \\
\tilde{A}^{P-1} \tilde{C} & \tilde{A}^{P-2} \tilde{C} & \ldots & \tilde{C}
\end{array}\right] \\
G=\left[\begin{array}{cccc}
\tilde{B} f_{N}(0) & \ldots \\
(\tilde{A} \tilde{B}-\tilde{B}) f_{1}(0)+\tilde{B} f_{1}(1) & (\tilde{A} \tilde{B}-\tilde{B}) f_{2}(0)+\tilde{B} f_{2}(1) & \ldots & (\tilde{A} \tilde{B}+\tilde{B}) f_{N}(0)+\tilde{B} f_{N}(1) \\
\vdots & \vdots & \vdots \\
\tilde{B} f_{1}(P-1)+ & \tilde{B} f_{2}(P-1)+ & \ldots & \tilde{B} f_{N}(P-1)+ \\
\sum_{k=1}^{P-1}\left(\tilde{A}^{k} \tilde{B}-\tilde{A}^{k-1} \tilde{B}\right) f_{1}(P-1-k) & \sum_{k=1}^{P-1}\left(\tilde{A}^{k} \tilde{B}-\tilde{A}^{k-1} \tilde{B}\right) f_{2}(P-1-k) & \ldots & \sum_{k=1}^{P-1}\left(\tilde{A}^{k} \tilde{B}-\tilde{A}^{k-1} \tilde{B}\right) f_{N}(P-1-k)
\end{array}\right]
\end{array}
$$

\section{REFERENCES}

[1] Haitao Zhang, Zonghai Chen, Yongji Wang, Ming Li \& Ting Qin, (2006) “Adaptive predictive control algorithm based on Laguerre Functional Model", Int. J. Adapt. Control Signal Process, No. 20, pp 53-76.

[2] Mingzhu Xu, Heping Liu, Xiaoli Li \& Shenshan Li, (2006) "A Steady Adaptive Predictive Functional Control with Chaotic Optimization", Proceedings of the Sixth International Conference on Intelligent Systems Design and Applications (ISDA'06).

[3] Z. Ridong \& W. Shuqing, (2007) "Predictive Functional Controller with a Similar Proportional Integral Optimal Regulator Structure: Comparison with Traditional Predictive Functional Controller and Application to Heavy Oil Coking Equipment", Chin. J. Chem. Eng., Vol.15, No. 2, pp 247-253.

[4] X. J. Liu \& C. W. Chan., (December 2006) "Neuro-Fuzzy Generalized Predictive Control of Boiler Steam Temperature", IEEE Transactions on Energy Conversion, Vol. 21, No.4, pp 900-908. 
[5] Dong-Na Shi, Guo Peng \&Teng-Fei Li., (12-15 July 2008) "Gray Predictive Adaptive Smith-PID Control and Its Application", Proceedings of the Seventh International Conference on Machine Learning and Cybernetics, Kunming, pp 1980-1984.

[6] Keming Xie, Wei Hao \& Jun Xie., (2007) "Superheated Steam Temperature Cascade Control System Based on Fuzzy-Immune PID”, Fourth International Conference on Fuzzy Systems and Knowledge Discovery (FSKD 2007).

[7] Yingfeng Ji \& Yaoyu Li, (11-13 July 2007) "Orthonormal Basis Function Based Transient Modeling for Boring Tool Degradation Monitoring”, Proceedings of the 2007 American Control Conference Marriott Marquis Hotel at Times Square New York City, USA.

[8] J.Richalet \& D. O’Donovan, (2009) "Predictive Functional Control Principles and Industrial Applications", London, Springer.

[9] Liuping Wang, (2009) "Model Predictive Control System Design and Implementation Using MATLAB®", London, Springer.

[10] X. Mingzhu, L. Shenshan \& X. Zhiping, (June 25 - 27 2008) "Predictive Functional Control on Kautz with Genetic Optimization", Proceedings of the 7th World Congress on Intelligent Control and Automation, Chongqing, China.

[11] Mingzhu Xu, Yiping Jiang, Jie Wen \& Cunzhi Pan, (July 6-9 2010) "Multiform Optimization of Predictive Functional Control Based on Kautz Model", Proceedings of the 8th World Congress on Intelligent Control and Automation, Jinan, China.

[12] A. Mbarek, H. Messaoud \& G. Favier, (2003) "Robust Predictive Control Using Kautz Model" ICECS.

[13] Mingzhu Xu, Xiaoli Li, HepingLiu \& Yanshuang Hao, (20 - 23 August 2006) "Adaptive Predictive Functional Control with Stochastic Search", Proceedings of the 2006 IEEE International Conference on Information Acquisition, Weihai, Shandong, China.

[14] Liuping Wang, (2004) "Discrete model predictive controller design using Laguerre functions", Journal of Process Control, Vol. 14, pp 131-142.

\section{Authors}

\section{Reza Dadkhah Tehrani}

He was born in Isfahan, Iran in March 1986. He received the Bachelor of Science degree in Electronic Engineering in 2008 from Shahid Rajaee University of Tehran and the Master of Science degree in Control Engineering in 2011 from Malek Ashtar University of Technology of Tehran. He works as an Instrumentation and Control Engineer in Arianir Co. (Engineering Power Company) and as a Research Assistant at Malek Ashtar University of Technology. His research interest includes adaptive control, system identification and predictive control.

\section{Mohammad Hossein Ferdowsi}

He received the B.Sc. and M.Sc. degrees in Electrical Engineering from Sharif University of Technology and Ph.D. degree in Electrical Engineering from University of Tehran in 1977, 1980 and 2004 respectively. He has worked in industry for five years as an Instrumentation and Control Engineer. From1985 to 1987 he has been a lecturer at Sharif University of technology and from1987 up to now he has been at Malek Ashtar University of Technology as a faculty member. His research interest is in multivariable and adaptive control systems, intelligent systems and target tracking.
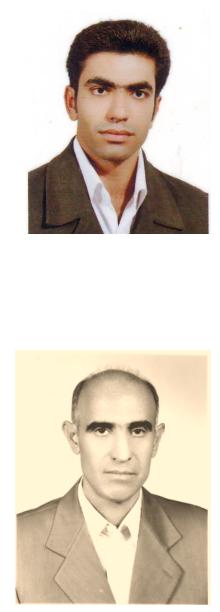